\title{
Interpolant based Decision Procedure for Quantifier-Free Presburger Arithmetic
}

\author{
Shuvendu K. Lahiri \\ Microsoft Research, Redmond \\ Krishna K. Mehra \\ Microsoft Research India, Bangalore
}

shuvendu@microsoft.com

kmehra@microsoft.com

\begin{abstract}
Recently, off-the-shelf Boolean SAT solvers have been used to construct ground decision procedures for various theories, including Quantifier-Free Presburger (QFP) arithmetic. One such approach (often called the eager approach) is based on a satisfiability-preserving translation to a Boolean formula. Eager approaches are usually based on encoding integers as bit-vectors and suffer from the loss of structure and sometime very large size for the bit-vectors.

In this paper, we present a decision procedure for QFP that is based on alternately under and over-approximating a formula, where Boolean interpolants are used to compute the overapproximation. The novelty of the approach lies in using information from each phase (either underapproximation or overapproximation) to improve the other phase. Our preliminary experiments indicate that the algorithm consistently outperforms approaches based on eager and very lazy methods, on a set of verification benchmarks. In our experience, the use of interpolants results in better abstractions being generated compared to an earlier method based on proofs directly.
\end{abstract}

KEYWORDS: SAT-solver, interpolants, decision procedures, linear arithmetic, satisfiability modulo theories

Submitted November 2006; revised April 2007; published May 2007

\section{Introduction}

Decision procedures for quantifier-free theories are the cornerstones of many automated analysis tools for software and hardware. Software verification tools like SLAM [3] use decision procedures to perform automated predicate abstraction and refinement of programs; ESC-JAVA [14] uses decision procedures to discharge verification conditions in static analysis of programs. High-level hardware verification tools including UCLID [6] use decision procedures for checking first-order formulas arising from bounded model checking or invariant checking for models of microprocessor and cache coherence protocols.

The quantifier-free queries that arise in verification typically have a lot of Boolean structure in addition to theory constraints. This requires an interplay between a search algorithm to case split on the Boolean structure and a decision procedure for the ground theory. In recent years, several approaches have emerged to leverage the rapid improvements in Boolean Satisfiability (SAT) solving [24]. These techniques differ mainly in how closely 
the SAT solver interacts with theory reasoning. The eager approaches rely on translating the quantifier-free formula to an equisatisfiable Boolean formula and use a SAT solver to solve the resultant Boolean formula [6,33]. The (very) lazy techniques create a Boolean abstraction of the first-order formula, and refine it based on Boolean assignments that are inconsistent with the underlying theory $[1,4,13]$. Both these approaches treat the SATsolver as a black-box. More recently, the DPLL(T) [25] based approaches have augmented the Davis, Putnam, Logeman and Loveland (DPLL) [9, 10] algorithm for the Boolean SAT solvers with theory specific reasoning $[25,5] .{ }^{1 .}$

Quantifier-free Presburger (QFP) arithmetic is the quantifier-free fragment of Presburger arithmetic [27], which deals with linear arithmetic constraints along with Boolean connectives. This fragment is useful for modeling most arithmetic constraints that arise in program and hardware verification $[12,2,30,6]$.

Kroening et al. [20] proposed a framework for integrating the Boolean and the theory reasoning for QFP, based on alternately under and over-approximating the input QFP formula. The algorithm searches for a satisfying solution in a sequence of increasingly large domains, lazily increasing the domain size when no satisfying solution is found in a smaller domain. The proof of unsatisfiability in a domain is used to construct an abstraction of the original formula. The abstractions are checked using a decision procedure for QFP. They illustrate that the procedure terminates and therefore constitutes a decision procedure for QFP.

In this work, we present an alternate implementation of the above framework that is based on Craig's interpolants [8,28] to construct the abstraction, once a formula is unsatisfiable in a given domain. The main difference between the two approaches lies in constructing an abstraction of an input QFP formula, once the formula is found unsatisfiable in a given domain. We believe the use of interpolants provides a much more semantic abstraction of the input formula that tries to abstract the proof of unsatisfiability from the particular domain. Although this is also the goal of the proof-based approach of Kroening et al., the abstractions generated can be heavily influenced by the structure of the input formula; the abstraction depends not only on the proof of unsatisfiability in a given domain, but also on maps that depend on the structure of the input formula and design decisions during implementation. Since these maps are not unique, the quality of the abstractions can vary.

The algorithm we propose in this paper also differs from the algorithm of Kroening et al. in other respects. First, we do not require a complete decision procedure for QFP to check the abstraction. Secondly, we leverage the learning from the search in the smaller domains when moving to a larger domain. We compare our approach with Kroening et al.'s work in detail in Section 5.

At a high level, our algorithm works as follows: consider a QFP formula $\phi$. Given a domain $D$, it is possible to construct a Boolean formula $\phi_{u}$ whose satisfiability determines the existence of a satisfying solution for $\phi$ in the domain $D$. This Boolean formula underapproximates the original formula $\phi$. If $\phi_{u}$ is unsatisfiable, then we use Boolean interpolant generation to construct a formula $\phi_{o}$ in $\mathrm{QFP}$ that is an overapproximation of $\phi$ (we de-

1. Although the latter techniques are also referred to as lazy techniques, we mostly refer to the very lazy techniques $[1,4,13]$ that treat the SAT-solver as a black box as lazy in this paper. 
fer the actual details of the procedure to Section 3). Intuitively, the overapproximation abstracts the reason why $\phi$ was unsatisfiable in the particular domain $D$, in terms of the constraints in $\phi$. The generated overapproximation of $\phi_{o}$ serves two purposes: first, if $\phi_{o}$ is unsatisfiable, then $\phi$ is unsatisfiable and secondly, we can generate some conflict clauses (tautologies in QFP) from $\phi_{o}$ that can be added to $\phi$, to prune the search space for future underapproximations.

The theory of QFP has a small-model property for any formula $\phi$ in the theory, i.e., if $\phi$ is satisfiable, then it has a satisfying assignment in a finite domain $D_{\max }$ determined by the formula $\phi$. This ensures that we can start with a small domain $D$ and increase the size lazily until the maximum domain $D_{\max }$ is reached. The small-model property also allows us to use an incomplete decision procedure for QFP to check the satisfiability of $\phi_{o}$. In our experience, the maximum domain size $D_{\max }$ is almost never reached.

We have implemented the algorithm in Zap theorem prover [34] and provide preliminary experimental evaluation of the approach on a set of verification benchmarks. The algorithm seems to consistently outperform an implementation based on purely eager encoding (that use the maximum domain size $D_{\max }$ to encode an equisatisfiable formula to SAT). It also outperforms an implementation of the very lazy Verifun [13] approach on these benchmarks. We have also implemented a variant of Kroening et al.'s approach inside Zap, to evaluate the quality of abstractions generated by the interpolant-based method and the proof-based method. Our preliminary evaluation indicates that the interpolant-based approach constructs much more concise abstractions, which result in improved runtime.

The rest of the paper is organized as follows: In Section 2, we describe the background material including eager and lazy approaches for leveraging SAT solvers. We also describe basics of interpolants. In Section 3, we motivate the algorithm and present the details of the decision procedure for QFP. Section 4 describes the experimental evaluation of the approach. We describe related work in Section 5 and finally conclude. The Appendix presents a variation of the algorithm that does not require computing $D_{\max }$ for completeness.

\section{Preliminaries}

In this section, we provide some background on the logic QFP, eager and the (very) lazy approaches for solving QFP along with Boolean interpolants.

\subsection{Quantifier-free Presburger (QFP) Arithmetic}

Presburger arithmetic is the first-order theory of structure $\langle\mathbb{N}, 0,1,+, \leq\rangle$, where $\mathbb{N}$ denote the set of natural numbers. Since every integer variable can be expressed as the difference of two natural numbers, we assume that the underlying domain is the set of integers $\mathbb{Z}$. Quantifier-free Presburger Arithmetic (QFP) is the quantifier-free fragment of Presburger arithmetic. Let $X$ be a set of integer variables. An atomic formula (atomic-formula) in this theory (also refered to as a linear constraint) is an expression of the form:

$$
\begin{aligned}
& \sum_{i} a_{i} * x_{i} \leq c, \\
& \text { ग5त्ताप }
\end{aligned}
$$


where $x_{i} \in X$ and the coefficients $a_{i}$ and $c$ are constants in $\mathbb{Z}$. A formula in this QFP is a Boolean combination of atomic formulas:

$$
\begin{aligned}
\text { formula }::= & \text { true } \mid \text { false } \mid \text { atomic-formula } \\
\mid & \text { formula } \wedge \text { formula } \mid \text { formula } \vee \text { formula } \mid \neg \text { formula }
\end{aligned}
$$

Observe that the other relational operators $\{=, \neq,<,>, \geq\}$ can be expressed in QFP.

A formula $\phi$ in QFP is satisfiable if there is an assignment $\rho: X \rightarrow \mathbb{Z}$ that maps each $x_{i} \in X$ to an integer value, such that the evaluation of $\phi$ under $\rho$ is true. A formula $\phi$ is unsatisfiable if there is no assignment $\rho$ under which $\phi$ evaluates to true.

A monome is a conjunction of atomic formulas and their negation in QFP. Checking the satisfiability of a monome in QFP is NP-complete [26]. However, efficient algorithms based on branch-and-bound heuristics are implemented in various Integer Linear Programming (ILP) solvers like LP_SOLVE [22] and commercial tools like CPLEX [17] to solve this fragment. The complexity of checking the satisfiability of QFP formulas is no worse than checking satisfiability of a conjunction of atomic formulas. Previous studies have indicated that ILP solvers do not perform well for QFP formulas with a significant Boolean structure, that arise from verification problems [30,20]. To circumvent this problem, two methods have been proposed in recent years to leverage backtracking search of modern Boolean satisfiability solvers. We describe the two approaches in the next section.

\subsection{Lazy and Eager Methods for solving QFP}

\subsubsection{LAZY METHODS}

The lazy methods [1, 29, 4] leverage the backtracking of modern Boolean Satisfiability (SAT) solvers to case-split on the Boolean structure in the QFP and use a ILP solver to check the satisfiability of a conjunction of linear constraints. The algorithms can be loosely described as follows:

1. The QFP formula $\phi$ is abstracted to a Boolean formula $\phi_{a}$ by replacing each atomic constraint with a Boolean variable.

2. The SAT solver enumerates satisfying solutions over $\phi_{a}$ and uses the ILP solver to validate the solution over the $\mathrm{QFP}$ theory.

3. If the satisfying solution is consistent with the QFP theory, the procedure returns satisfiable. Otherwise, the solver returns a conflict clause (a tautology in QFP) that rules out the current assignment. Typically, the literals that appear in the proof of unsatisfiability of the current assignment, constitute the conflict clause $[4,13]$. This helps towards finding a "minimal" unsatisfiable core to rule out more than just the present satisfying assignment. The conflict clause is added to $\phi_{a}$ and Step 2 is repeated.

It must be clarified here that the method described above belongs to the very lazy class wherein the theory solvers validate total models returned by the SAT solver. The SAT solver needs to be restarted from scratch after adding the conflict clause. 


\subsubsection{EAGER Methods}

Eager methods (e.g. implemented in UCLID [21]) are based on translating a QFP formula $\phi$ to an equisatisfiable Boolean formula $\phi_{\text {bool }}$, such that $\phi$ is satisfiable if and only if $\phi_{\text {bool }}$ is satisfiable. QFP enjoys a small-model property - a QFP formula $\phi$ is satisfiable over $\mathbb{Z}$ if and only if it is satisfiable over a finite domain $\mathbb{D} \subseteq \mathbb{Z}$. The measure $\log (\mathbb{D})$ denotes the number of Boolean variables to represent the domain $\mathbb{D}$. This means that if a model for the formula exists, it can be found by encoding each variable in $\log (\mathbb{D})$ bits.

Let $m$ be the number of atomic formulas in $\phi$ and $n$ be the number of variables in $X$. When the set of atomic constraints in $\phi$ is restricted to difference logic constraints (where the atomic formulas are restricted to $x_{i}-x_{j} \leq c$ ), the size of the domain $\log (\mathbb{D})$ is $O\left(\log (n)+\log \left(c_{\max }\right)\right)$, where $c_{\max }$ is the absolute value of largest constant $c$ that appears in any of the constraints. Seshia and Bryant [30] show that for general linear constraints, $\log (\mathbb{D})$ is bound by

$$
\log (n)+\log (m)+\log \left(c_{\max }\right)+k *\left(\log \left(a_{\max }\right)+\log (w)\right),
$$

where $a_{\max }$ is the maximum absolute value of any coefficient, $k$ is the number of nondifference atomic formulas in $\phi$ and $w$ is the maximum number of variables in any linear constraint. When the number of non-difference constraints in $\phi$ is small, the size of $\log (\mathbb{D})$ is almost logarithmic in $n$ and $m$. The logarithms above are in base 2 .

The above bounds suggest that one can encode each variable $x \in X$ using $\log (\mathbb{D})$ Boolean variables and translate $\phi$ to a Boolean formula. The arithmetic operator + can be encoded as an word adder, $*$ is implemented as a shift operator since variables are multiplied only with constants. Similarly, the relational operator $\leq$ is encoded as word comparator. The size of the resultant Boolean formula $\phi_{\text {bool }}$ incur a polynomial blowup (typically $\log (\mathbb{D})$ ) over $\phi$. Finally, the resultant formula can be checked using any state-of-the-art SAT solvers. We refer to this encoding as small-model encoding of a first-order formula.

\subsubsection{Comparison of THE TWO Methods}

In this section, we highlight the main weaknesses of the eager and the lazy approaches, that limit the scalability of the approaches:

The appeal of small-model based encoding to SAT lies in the fact that it provides only a polynomial blowup when translating a linear arithmetic formula to a Boolean formula. However, the blowup can be linear in the size of the number of constraints and variables in the first-order formula, when the number of non-difference constraints is large. The small model size also explodes in the presence of large constants in the formula. More seriously, small-model encoding suffer from a loss of structure of the formula. For example, consider the simple formula:

$$
\phi=x \leq y \wedge y \leq z \wedge z<x
$$

There is a polynomial algorithm for deciding the satisfiability of such conjunction of linear arithmetic formulas, based on negative cycle detection [7]. However, converting to a Boolean formula requires encoding of relational operators such as $\leq$ as a circuit and this introduces a lot of disjunctions in the boolean formula. The encoding of $\leq,+,=$ and other operators 
is quite standard and has not been discussed in this paper. This results in SAT solver to perform a lot of case splits before detecting unsatisfiability.

The lazy approaches (e.g. Verifun [13]) suffer from the need to invoke a decision procedure for the first-order theory a very large number of times in the presence of a lot of Boolean structure (i.e. disjunctions) in the formula. Moreover, since the decision procedures take over only after the SAT solver has found a Boolean model, the monome handed to the theory can have a lot of theory literals. When the monome is unsatisfiable in the theory, it often happens because of a very small subset of literals in the monome. Although the decision procedure can figure out the core reason for unsatisfiability (using the proof of unsatisfiability), the decision procedure is often overwhelmed with the size of the monome that it obtains from the SAT solver. This is particularly problematic for the theory of integer linear arithmetic, for which the decision procedures have exponential worst-case complexity.

\subsection{Boolean Interpolants}

Consider two satisfiable Boolean formulas $A$ and $B$ such that $A \wedge B$ is unsatisfiable. Let $V$ be the set of Boolean variables shared by both $A$ and $B$. An (Boolean) interpolant [8] of the pair of formulas $(A, B)$ is a Boolean formula $I$, such that:

1. $A \Rightarrow I$,

2. $I \wedge B$ is unsatisfiable, and

3. The set of variables in $I$ is a subset of $V$.

Pudlak [28] showed that given the proof of unsatisfiability of $A \wedge B$, an interpolant $I$ can be obtained in time linear to the size of the proof. A description of the algorithm when both $A$ and $B$ are present in conjunctive normal form (CNF) has been described by McMillan [23]. The motivation for using the interpolant $I$ of $(A, B)$ is usually two-fold: (a) it is an abstraction of $A$ that is sufficient to prove the unsatisfiability with $B$ and (b) it is over the common variables of $A$ and $B$.

\section{Interpolant-based Decision Procedure for QFP}

In this section, we describe an algorithm to decide the satisfiability of a QFP formula $\phi$. The algorithm alternates between two phases that check the satisfiability of an underapproximation and overapproximation of $\phi$, respectively. In the underapproximation phase, the algorithm creates a formula $\phi_{u}$ that is an underapproximation of $\phi$ by restricting the domain of each variable that appear in the formula. If the formula $\phi_{u}$ has a satisfying assignment within the small bounds, it reports satisfiable. Otherwise, it computes an abstraction $\phi_{o}$ of $\phi$ by using Boolean interpolants. The abstract formula $\phi_{o}$ is then checked for (un) satisfiability. If $\phi_{o}$ is unsatisfiable, then $\phi$ is unsatisfiable. Otherwise, we repeat the phases with an increased domain for the variables. The algorithm adds additional clauses that it discovers while checking $\phi_{o}$ to the formula $\phi$, to speed up the underapproximation phase in the further iterations. We describe the algorithm in details in the next few paragraphs. 
1. [Input]. Given a QFP formula $\phi$.

2. [Encoding]. Construct the Boolean structure of $\phi$ by replacing every atomic formula $e$ with a fresh Boolean variable $b_{e}$ in $\phi$. The resultant formula $\phi_{b}$ will be referred to as the (Boolean) skeleton of $\phi$. Let atoms $(() \phi)$ denote the set of atomic formulas in $\phi$. We introduce a set of fresh variables $B=\left\{b_{e} \mid e \in \operatorname{atoms}(\phi)\right\}$ and create a formula

$$
\phi_{t h}=\left(\bigwedge_{e \in a t o m s(\phi)} b_{e} \Leftrightarrow e\right)
$$

called the theory portion of $\phi$. Finally, the formula representing the conjunction of the Boolean skeleton and the theory component is called $\phi_{b t}$ :

$$
\phi_{b t} \doteq \phi_{b} \wedge \phi_{t h}
$$

3. [Initialize]. Compute the maximum model size $D_{\max }$ for the variables using Equation 1. The initial domain for each variable is restricted to a small number $D$ (say 2), i.e. each variable $v \in \operatorname{vars}(\phi)$ is such that $-D<v \leq D$.

4. [Boolean UNSAT]. We first check if the skeleton of $\phi_{b t}$ is unsatisfiable using the SAT solver. If $\phi_{b}$ is unsatisfiable, the algorithm returns UNSATISFIABLE.

5. [Underapproximation]. We construct a Boolean formula for $\phi_{t h}$ by using the smallmodel encoding technique described in Section 2.2.2. We use $B E\left(\phi_{t h}, D\right)$ to denote the Boolean translation. Let the resultant Boolean formula be $\phi_{u}$, an underapproximation of $\phi_{b t}$ :

$$
\phi_{u} \doteq \phi_{b} \wedge B E\left(\phi_{t h}, D\right)
$$

We check if $\phi_{u}$ is satisfiable using a SAT solver. If $\phi_{u}$ is satisfiable, the algorithm returns SATISFIABLE. If $\phi_{u}$ is unsatisfiable and $D \geq D_{\max }$, we return UNSATISFIABLE.

6. [Overapproximation]. If $\phi_{u}$ is unsatisfiable, compute the Boolean interpolant $\phi_{I}$ of $\phi_{b}$ and $B E\left(\phi_{t h}, D\right)$. We construct the formula $\phi_{o}$ which is an overapproximation of $\phi_{b t}$ as follows:

$$
\phi_{o} \doteq \phi_{I} \wedge \phi_{t h}
$$

We check the satisfiability of $\phi_{o}$ using a conflict clause generator $C C G()$ for QFP (described later in this section). If $C C G\left(\phi_{o}\right)$ returns UNSATISFIABLE, the algorithm returns UNSATISFIABLE. Otherwise, $C C G\left(\phi_{o}\right)$ returns a set of (conflict) clauses $\phi_{c}$, representing tautologies in the theory of QFP.

We augment the conflict clauses $\phi_{c}$ to $\phi_{b}$ to create a more constrained Boolean skeleton of $\phi_{b t}$ :

$$
\phi_{b} \leftarrow \phi_{b} \wedge \phi_{c}
$$


7. [Repeat]. Increase the bound $D$ for each variable by some predetermined amount $\delta>0$, i.e. $D=D+\delta$. Goto step 4 .

Let us first observe a couple of points about this algorithm.

- When $\phi_{u}$ is unsatisfiable in step 5 , we know that the two components of $\phi_{u}$, namely $\phi_{b}$ and $B E\left(\phi_{t h}, D\right)$ are each satisfiable in isolation. This is because step 4 ensures that $\phi_{b}$ is satisfiable and $B E\left(\phi_{t h}, D\right)$ is always satisfiable for any domain.

- The common variables in $\phi_{b}$ and $B E\left(\phi_{t h}, D\right)$ are only the variables from $B$. This allows us to construct an interpolant in terms of the $B$ variables, independent of the integer variables or the variables introduced during translating an integer variable to a symbolic bit vector.

\subsection{Conflict Clause Generator ( $C C G$ )}

The conflict clause generator algorithm takes as input the QFP formula $\phi_{o} \doteq \phi_{I} \wedge \phi_{\text {th }}$, where $\phi_{I}$ is a Boolean formula over $B$ variables and checks for the unsatisfiability of the formula:

- If it returns UNSATISFIABLE, then the formula $\phi_{o}$ is unsatisfiable.

- Otherwise, it returns a conjunction of clauses $\phi_{c} \doteq \bigwedge_{i} c_{i}$, such that $\phi_{o} \Longrightarrow \phi_{c}$ and each $c_{i}$ is a disjunction of literals over $B$.

The conflict clauses generator can be implemented as a simple variation of a lazy SATbased theorem proving framework as follows: Initially, $\phi_{c}$ is assigned true. The SAT solver enumerates assignments to $B$ variables that satisfy $\phi_{I}$ and checks if the assignment satisfies $\phi_{\text {th }}$ using a (possibly incomplete) decision procedure for a conjunction of linear arithmetic constraints.

If the assignment is found unsatisfiable by the linear arithmetic decision procedure, the decision procedure returns a "proof core", a subset of constraints that are inconsistent. The negation of the proof core is a clause $c_{i}$ that is added to $\phi_{c}$ (after mapping an atomic expression $e$ to the corresponding $b_{e}$ variable). The clause $c_{i}$ is also added to the $\phi_{I}$ to prevent it from generating the same assignment. However, if the linear arithmetic decision procedure returns satisfiable, we simply add the negation of the assignment to $\phi_{I}$ and repeat the loop. The process is continued until the number of satisfying assignments (that are also satisfiable in the linear arithmetic theory) does not exceed some threshold. We currently limit this threshold to be 5 , i.e. after obtaining more than 5 satisfying solutions, we return $\phi_{c}$.

The usefulness of the conflict clause generator comes from the fact that it adds some structure back to the Boolean formula $\phi_{u}$. The conflict clauses added aid the SAT-solver to avoid some case-splits when it is checking the satisfiability of the formula $\phi_{u}$ in the subsequent iterations with larger $D$ values. 


\subsection{Correctness}

It is not hard to show that the algorithm is sound and complete. The algorithm returns with SATISFIABLE or UNSATISFIABLE in steps 4, 5, and 6. We use the following invariant on the algorithm:

Lemma 1. At any point in the algorithm, $\phi_{b t}$ is equisatisfiable with $\phi$. In step $5, \phi_{u} \Longrightarrow$ $\phi_{b t}$, and in step $6, \phi_{b t} \Longrightarrow \phi_{o}$.

Proof. The proof follows simply by induction on the number of iterations of the algorithm.

For the first iteration, $\phi_{b t}$ is equisatisfiable with $\phi$ since $\phi=\exists B: \phi_{b t}$. Since $\phi_{u}$ simply restricts the domain of each variable in $\phi_{b t}$, clearly $\phi_{u} \Longrightarrow \phi_{b t}$. In step 6, assume $\phi_{u}$ is unsatisfiable. Recall that $\phi_{b}$ is not unsatisfiable because we have ensured that in step 4 . We also know that the formula $B E\left(\phi_{t h}, D\right)$ is satisfiable. This is because it just says that the value of $b_{e}$ is the same as the value of $e$. There are no constraints on any $b_{e}$ variables or the atomic constraints $e$. This explains the reason why we have step 4 explicitly in the algorithm. Moreover, recall that $\phi_{u}$ is a Boolean formula. Therefore, we can construct a Boolean interpolant $\phi_{I}$ for the pair $\left(\phi_{b}, B E\left(\phi_{t h}, D\right)\right)$. Since $\phi_{b} \Longrightarrow \phi_{I}, \phi_{b t} \Longrightarrow \phi_{o}$.

Now, let us assume that the lemma holds for some iteration. We want to prove that it holds for the next iteration. Recall, that the only step in which $\phi_{b}$ changes is in step 6 . We also know that $\phi_{o} \Longrightarrow \phi_{c}$ (from the property of the conflict clause generator), and hence $\phi_{b t} \Longrightarrow \phi_{c}$. This in turn implies that $\phi_{b t} \Longrightarrow\left(\phi_{b t} \wedge \phi_{c}\right)$. Moreover, $\left(\phi_{c} \wedge \phi_{b t} \Longrightarrow \phi_{b t}\right)$. Hence $\left(\phi_{c} \wedge \phi_{b t}\right) \Leftrightarrow \phi_{b t}$. Therefore, the formula $\phi_{b t}$ is equivalent across all iterations steps. This preserves other parts of the lemma.

Theorem 1. The algorithm described above is sound and complete for QFP.

Proof. It is easy to see that the algorithm terminates, since $D$ is monotonically increased and finally crosses $D_{\max }$. Observe that the algorithm returns SATISFIABLE, if and only when it finds $\phi_{u}$ SATISFIABLE. Lemma 1 ensures soundness of the algorithm. The algorithm returns UNSATISFIABLE if and only if a formula weaker than $\phi_{b t}$ is unsatisfiable. The weaker formulas in step 4 , step 5 and step 6 are $\phi_{b}, \phi_{u}$ (only when $D \geq D_{\max }$ ) and $\phi_{o}$ respectively.

\subsection{Example}

In this section, we illustrate the working of the algorithm on a simple example. Consider the following formula $\phi$ :

$$
\begin{aligned}
\phi \doteq & (x<y) \wedge(y<z) \wedge(z<w \vee z<x) \wedge \\
& (\neg(z<w) \vee x<2 * w+1) \wedge(\neg(z<w) \vee \neg(x<2 * w+1))
\end{aligned}
$$

Let us introduce Boolean variables $\left\{b_{x<y}, b_{y<z}, b_{z<w}, \ldots\right\}$ for the atomic constraints in $\phi$. Let $\phi_{t h}$ denote the constraint $\left[\bigwedge_{e} b_{e} \Leftrightarrow e\right]$ as before and $\phi_{b}$ be the Boolean skeleton of $\phi$.

Let us start with a domain $D$ where each variable takes values in $\{0,1\}$. The underapproximation of $\phi$ created by restricting each variable to $D$ is unsatisfiable. Let

$$
\phi_{I}=\left(b_{x<y} \wedge b_{y<z} \wedge\left(b_{z<w} \vee b_{z<x}\right)\right)
$$


be the interpolant generated in step 6 of the algorithm. In this domain, the formula $(x<y \wedge y<z \wedge(z<w \vee z<x))$ is unsatisfiable. The monome $x<y \wedge y<z \wedge z<w$ can't be satisfied because each variable can only take two values, and the monome $x<$ $y \wedge y<z \wedge z<x$ is always unsatisfiable.

Now $\phi_{o} \doteq \phi_{I} \wedge \phi_{t h}$ is fed to the conflict clause generator. Even though $\phi_{o}$ is satisfiable, it generates a conflict clause

$$
\phi_{c} \doteq\left(\neg b_{x<y} \vee \neg b_{y<z} \vee \neg b_{z<x}\right)
$$

Once we add the conflict clause $\phi_{c}$ to $\phi_{b}$, we obtain unsatisfiable in the Boolean skeleton. This example illustrates the interesting case where the unsatisfiability of $\phi$ is detected in step 4 of the algorithm.

\subsection{Discussion}

In this section, we illustrated a decision procedure for QFP (or in general for a theory $T$ ) that uses a sound procedure for deciding a formula in QFP (or in theory $T$ ) as part of the conflict clause generator. The $C C G()$ decision procedure need not be complete for the theory. One can use a fast decision procedure for a subset of integer linear arithmetic (e.g. based on negative cycle detection algorithms), coupled with simple rules for more general arithmetic. This enables us to plug in any fast decision procedure for a subset of QFP (e.g. difference logic) as the $C C G()$. To ensure the completeness of the procedure, we need to compute the small-model size $D_{\max }$ for a formula in the theory and search this domain in the worst case.

However, we can avoid the need to compute the small-model size $D_{\max }$, if we have a sound and complete decision procedure for QFP as the conflict clause generator. In this case, one can ensure that the sequence of overapproximations $\phi_{o}^{1}, \phi_{o}^{2}, \ldots$, (where $\phi_{o}^{i}$ denotes the overapproximation generated during the iteration $i$ of the above algorithm) for the input formula $\phi$, will eventually converge to $\phi$. Details of such a decision procedure is described in the Appendix.

\section{Experiments}

We have implemented a prototype of the technique in the theorem prover Zap [34]. We compare the following four variants of eager and lazy decision procedure implemented in Zap. Since the C\# implementation of Zap and SharpSAT introduces some slowdown over other tools (e.g. Mathsat [5] or Barcelogic tools [25]), it is difficult to draw many conclusions about the techniques by comparing the results for their specific implementations. Hence, we evaluate the techniques by benchmarking against the following variants implemented in Zap:

Verifun: This is the implementation of the lazy proof-explicating theorem prover described in Section 2.2.1 based on the Verifun architecture [13]. The linear arithmetic is restricted to Unit Two Variable Per Inequality (UTVPI) constraints [18], for efficiency. We do not consider examples where Verifun returns satisfiable because of the incom- 
Interpolant based Decision Procedure for Quantifier-Free Presburger Arithmetic

pleteness of the procedure. The UTVPI decision procedure suffices to prove a lot of examples of our example set even in the presence of more linear arithmetic.

Eager. This implements a small-domain encoding of a quantifier-free formula to a Boolean formula [6] using the eager encoding technique mentioned in Section 2.2.2. For encoding QFP, it uses the small model size computed by Seshia et al. [30] reported in Equation 1.

EaZI. This is the implementation of the algorithm Eager Zap with Interpolants (EaZI) described in Section 3. The initial size of the domain is restricted to assign 3 bits for each integer variable. Each subsequent iteration increases the number of bits per variable by 2. For the $C C G()$ generation, it uses the Verifun procedure described above. Observe that since the Verifun procedure is restricted to UTVPI, $C C G()$ is not complete for general linear arithmetic.

Proof. This option implements a variant of the EaZI approach, where the only difference lies in how the overapproximation is generated from the proof of unsatisfiability in a given domain. The overapproximation phase uses the approach by Kroening et al. [20]; the proof of unsatisfiability from the SAT solver is used directly to select a subset of clauses in the input formula, instead of the use of interpolants in EaZI. The input formula has to be first converted to a clausal form, and a suitable mapping from each clause in the propositional formula (resulting from the small model encoding) to a clause in the original formula is maintained. More details of the mapping can be found in earlier work [20]. The main purpose of the comparison with this approach is to evaluate the quality of the abstractions generated by the two methods.

In all the variants, we used SharpSAT as the Boolean SAT solver. SharpSAT is a variant of ZChaff [24] developed by Lintao Zhang at Microsoft. We ran the experiments on a $3 \mathrm{GHz}$ machine running Windows with 1 GB of memory. A timeout of 300 seconds was set for each benchmark.

We have performed preliminary experiments on two sets of verification benchmarks: (1) Mathsat: This a set of QFP benchmarks obtained from the timed automata verification problems [1]. An analysis of the formulas in a previous study [11] suggests that the coefficients of the QFP formulas are restricted to $\{-1,0,1\}$ (with more than two variables though), with the number of integer variables ranging from around 10 to around 150. Most of the variables in these benchmarks are Boolean variables. (2) SAL: This set of benchmarks [11] consists of formulas derived from bounded model checking of linear and hybrid systems and from test-case generation for embedded systems. Most of the benchmarks have significant linear arithmetic constraints and the number of integer variables range from tens to hundreds of variables.

\subsection{Results}

In this section, we present the results of running the different approaches on the Mathsat and SAL benchmarks. We analyze the results for different options: 

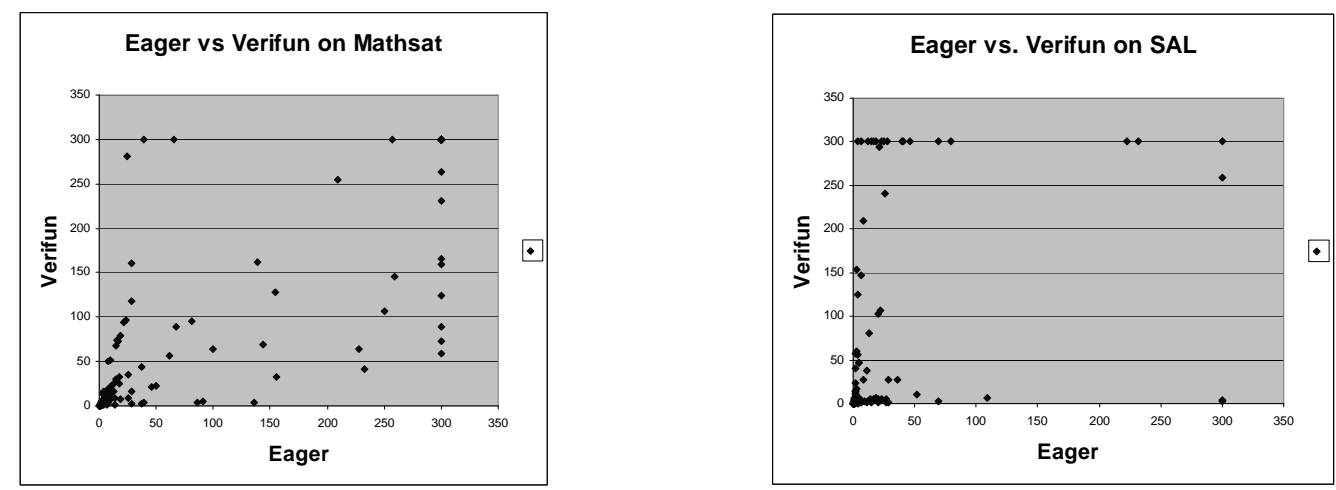

Figure 1. Eager vs. Verifun on Mathsat and Sal benchmarks.

\subsubsection{VERIFUn VS. EAGER}

Figure 1 compares the Verifun and the Eager approach on the benchmarks. Verifun scales better than Eager on the Mathsat benchmarks whereas Eager outperforms Verifun on SAL benchmarks. We believe the difference in performance of Verifun on the two sets of benchmarks can be explained by the number of times it invokes a theory decision procedure. Column 3 of Figure ?? (marked "\# Verifun Loops" for "Verifun") shows that the number of calls to the theory decision procedure for SAL benchmarks is at least an order greater than in the case for Mathsat benchmarks. The results suggest that neither Eager nor Verifun is robust enough for a large set of benchmarks.
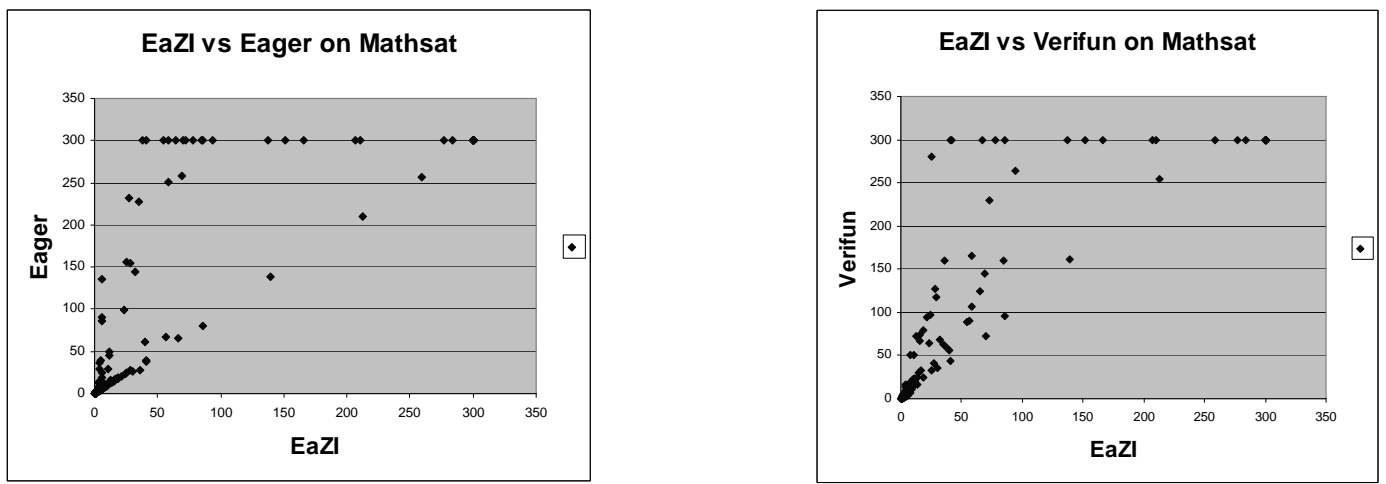

Figure 2. Eazi vs. Eager and Verifun on Mathsat Benchmarks.

\subsubsection{VERIFUn VS. EAZI}

Figure 2 and Figure 3 compares EaZI with Eager and Verifun on Mathsat and SAL benchmarks respectively.

EaZI outperforms Verifun consistently on both the set of benchmarks. To understand the improvement, we extracted some information for a subset of benchmarks. Table 1 compares 

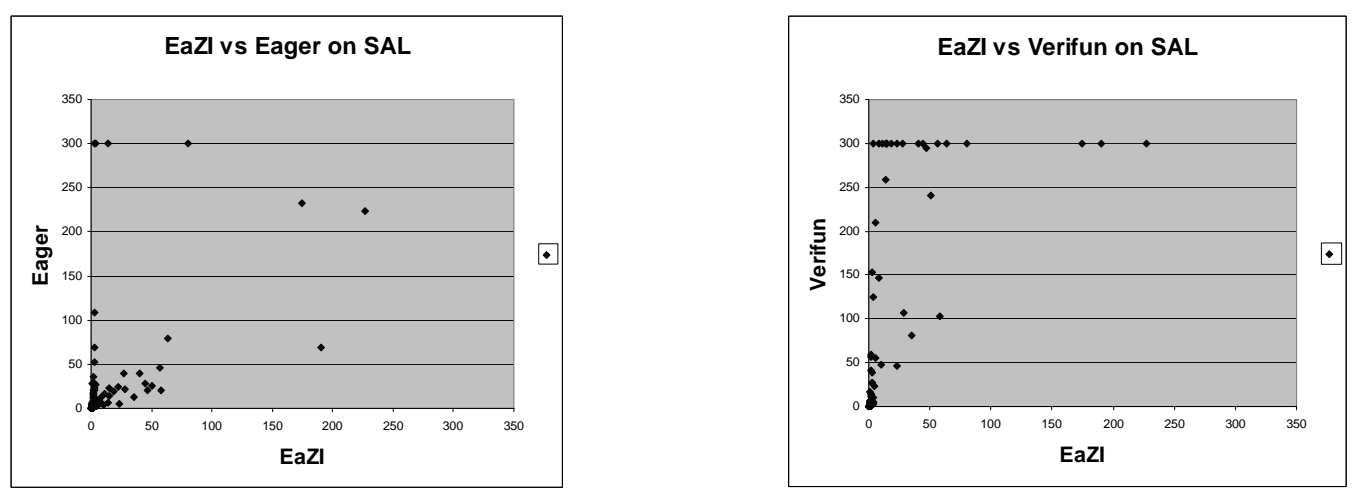

Figure 3. Eazi vs. Eager and Verifun on SAL Benchmarks.

Table 1. Analysis of EaZI vs. Verifun on Mathsat and SAL. A "TO" indicates a timeout of 300 seconds.

\begin{tabular}{|c|c|c|c|c|c|c|c|c|}
\hline Example & \multicolumn{2}{|c|}{ \# Verifun Loops } & \multicolumn{2}{c|}{ Avg. Monome size } & \multicolumn{2}{c|}{ Total theory time (secs) } & \multicolumn{2}{c|}{ Total time (secs) } \\
& Verifun & EaZI & Verifun & EaZI & Verifun & EaZI & Verifun & EaZI \\
\hline SAL & & & & & & & & \\
Carpark2-t1-4 & 200 & 2 & 755 & 1 & 222.27 & 0.06 & 259.13 & 26.31 \\
fischer3-mutex-5 & 372 & 72 & 399 & 66 & 116.84 & 2.45 & 153.70 & 8.84 \\
fischer6-mutex-3 & 143 & 18 & 426 & 19 & 39.28 & 0.17 & 57.19 & 2.92 \\
fischer9-mutex-3 & 221 & 40 & 603 & 15 & 107.14 & 0.25 & 146.44 & 8.45 \\
inf-bakery-invalid-10 & 180 & 0 & 211 & - & 47.90 & - & 59.25 & 3.45 \\
inf-bakery-mutex-9 & 426 & 66 & 200 & 38 & 149.77 & 1.11 & 210.02 & 5.04 \\
lpsat-goal-12 & 233 & 45 & 2019 & 19 & 87.75 & 0.34 & 294.49 & 92.64 \\
windowreal-safe2-4 & 254 & 8 & 328 & 23 & 19.14 & 0.17 & 38.04 & 3.00 \\
\hline MATHSAT & & & & & & & & \\
FISCHER10-5-fair & 19 & 3 & 969 & 49 & 30.46 & 0.34 & TO & 64.7 \\
FISCHER11-5-fair & 21 & 25 & 1072 & 113 & 42.54 & 11.53 & TO & 72.20 \\
FISCHER3-8-fair & 93 & 0 & 478 & - & 66.89 & - & 127.66 & 28.81 \\
FISCHER5-7-fair & 81 & 52 & 682 & 93 & 108.69 & 8.85 & TO & 94.46 \\
FISCHER8-6-fair & 36 & 40 & 935 & 108 & 94.72 & 14.29 & TO & 77.78 \\
PO3-9-PO3 & 38 & 3 & 618 & 20 & 25.53 & 0.07 & 250.58 & 94.32 \\
\hline
\end{tabular}

the two approaches in terms of three metric (a) number of times a theory decision procedure was invoked ("\# Verifun Loops"), (b) the average size of the number of constraints involved in the monome passed to the decision procedure ("Avg. Monome size"), (c) total time spent in the theory decision procedure ("Total theory time (secs)"), and (d) total time spent by the procedure ("Total time (secs)"). An example where "\# Verifun Loops" is 0 for the EaZI option (e.g. inf-bakery-invalid-10) corresponds to a satisfiable instance where EaZI found a satisfying assignment within the initial domain size (three bits for each variables). In these cases, the average monome size and the time spent in the theory decision procedures are not applicable.

We observed the following characteristics across a wide set of benchmarks: 
- The number of times a theory decision procedure is invoked is usually much smaller in the case of EaZI. This can be explained because the abstraction of the original formula $\phi_{o}$ has lot more conflicts in the Boolean structure and thus comes to theory less often.

- The size of monome and the total time spent in the theory is reduced at least by an order of magnitude in most cases. This is because the abstraction $\phi_{o}$ contains a lot fewer constraints than $\phi$.

- The main bottleneck of the EaZI method shifts from the theory decision procedure to SAT. In most cases, the interpolant generation consumes more than $80 \%$ of the total time. We believe the performance of our algorithm will further improve with improvement in the interpolant generation implementation in SharpSAT.

\subsubsection{EAGER Vs. EAZI}

Figure 2 and Figure 3 indicate that EaZI also outperforms the Eager method in most of the examples in this set.

The small model size for the most examples calculated by Equation 1 requires the Eager method to encode each variable with more than 15 bits in most case. In some cases with large number of non-difference constraints, the number of bits to encode each variable exceeds 100 bits. Although the EaZI method relies on the small-model size for completeness, it never reaches this maximum domain size for either proving unsatisfiability or satisfiability. For satisfiable instances, it finds a satisfying solution using a small number of bits for most examples. For unsatisfiable cases, the procedure exits from the $C C G()$ procedure with UNSATISFIABLE, or the conflict clauses generated from $C C G()$ makes the Boolean part of the formula $\phi_{b t}$ unsatisfiable. Even for the unsatisfiable instances, we need to increase the number of bits to at most 8 in most cases.

The Eager approach outperforms the EaZI approach on some of the SAL benchmarks. This is primarily because the number of bits to encode each variable is less than 20 and the overhead of computing interpolants in EaZI offsets the advantage of learning from smaller domain size.

\subsubsection{EAZI vs. Proof}

Figure 4 compares EaZI over the Proof [20] approach. The results indicate that the use of interpolants consistently outperforms our implementation of the proof-based method for constructing an abstraction of the input formula. Table 2 shows (on a representative set sampled) that the average size of the theory monomes and the number of verifun loops determine the relative efficiency of the two approaches.

We conjecture that the difference in the quality of abstractions generated in the two approaches can be partly explained by the "semantic" nature of the abstraction generated in the case of the interpolants. The proof-based approach can be seen as one particular heuristic to choose a subset of clauses from the original formula. The abstraction generated by the proof-based approach relies on the mapping from each clause in the propositional formula (generated after performing the small-model encoding) to a clause in the input 

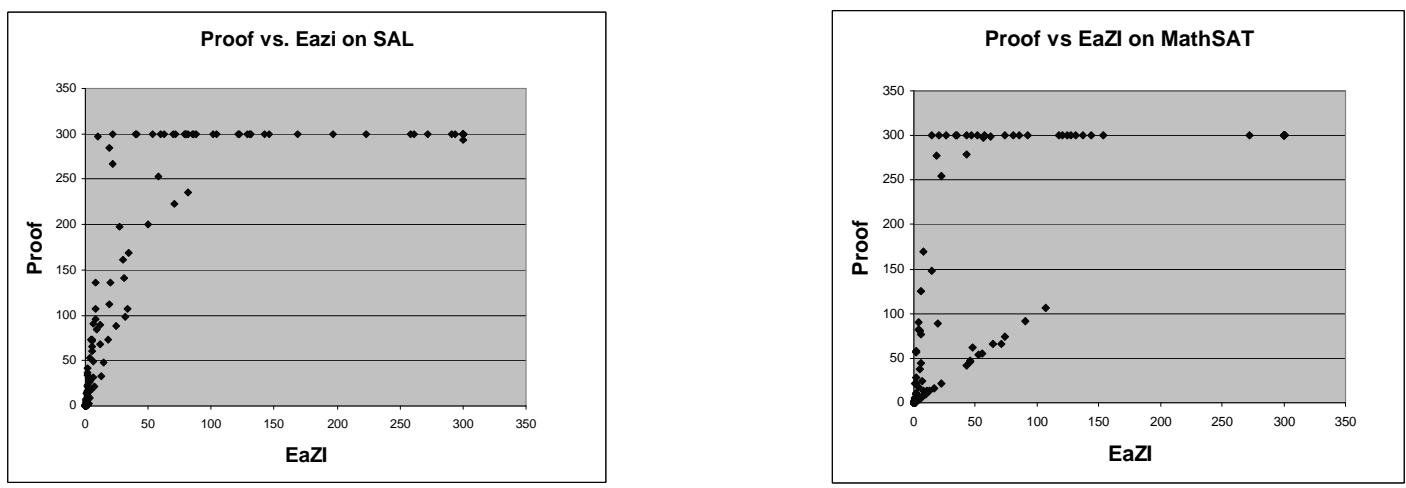

Figure 4. Comparing interpolant-based and proof-based abstractions.

Table 2. Analysis of EaZI vs. Proof on Mathsat and SAL.

\begin{tabular}{|c|c|c|c|c|c|c|c|}
\hline \multirow{2}{*}{ Example } & \multicolumn{2}{c|}{ Total time (secs) } & \multicolumn{2}{c|}{ \# Verifun Loops } & \multicolumn{2}{c|}{ Avg. Monome size } \\
& & Proof & EaZI & Proof & EaZI & Proof & EaZI \\
\hline \multirow{3}{*}{ sal } & fischer3-mutex7 & 267.21 & 37.38 & 1347 & 79 & 573 & 263 \\
& gasburner-prop3-5 & 88.76 & 10.35 & 47 & 38 & 151 & 28 \\
& infbakery-mutex-16 & 168.32 & 33.56 & 1021 & 295 & 386 & 78 \\
& pursuit-safety-5 & 235.82 & 65.18 & 563 & 186 & 368 & 115 \\
\hline mathsat & fischer5-6-fair & 292.93 & 64.03 & 24 & 3 & 564 & 39 \\
& po3-7-po3 & 57.98 & 2.33 & 3 & 3 & 460 & 19 \\
& fischer8-2-fair & 17.84 & 4.14 & 2 & 2 & 343 & 27 \\
\hline
\end{tabular}

formula. This mapping is not unique since a clause in the propositional formula can result from multiple clauses in the original formula. We have implemented the particular mapping scheme proposed by Kroening et al. to make the comparison fair. The use of auxiliary variables introduced during the CNF translation has the potential of introducing redundant clauses from the original formula in the abstraction. For the case of interpolants, there are two clear advantages (i) we do not need to start with a clausal representation of the input formula, and more importantly (ii) the abstraction generated only depends on the proof of unsatisfiability of the formula in a given domain. These factors make the EaZI approach more robust and less dependent on a particular encoding.

\subsubsection{Adding Conflict Clauses}

We conjectured that the advantage of EaZI over Eager comes from the fact that we add more structure (the conflict clauses from $C C G())$ to $\phi_{b}$ and thereby helps the SAT solver to prune away the search space efficiently. Figure 5 compares the EaZI approach with and without the conflict clauses from $C C G()$. The conflict clauses make marked difference in the results for the SAL benchmarks. Since the dominant time in the SAL experiments is in solving the Boolean formula $\phi_{u}$, addition of the conflict clauses help the SAT solver. However, adding the conflict clauses made almost no difference for the Mathsat examples. For most of the 
Mathsat examples, we found that the abstraction $\phi_{o}$ was proved unsatisfiable by the conflict clause generator after the first iteration. Hence the conflict clauses do not play a part in most of these examples.
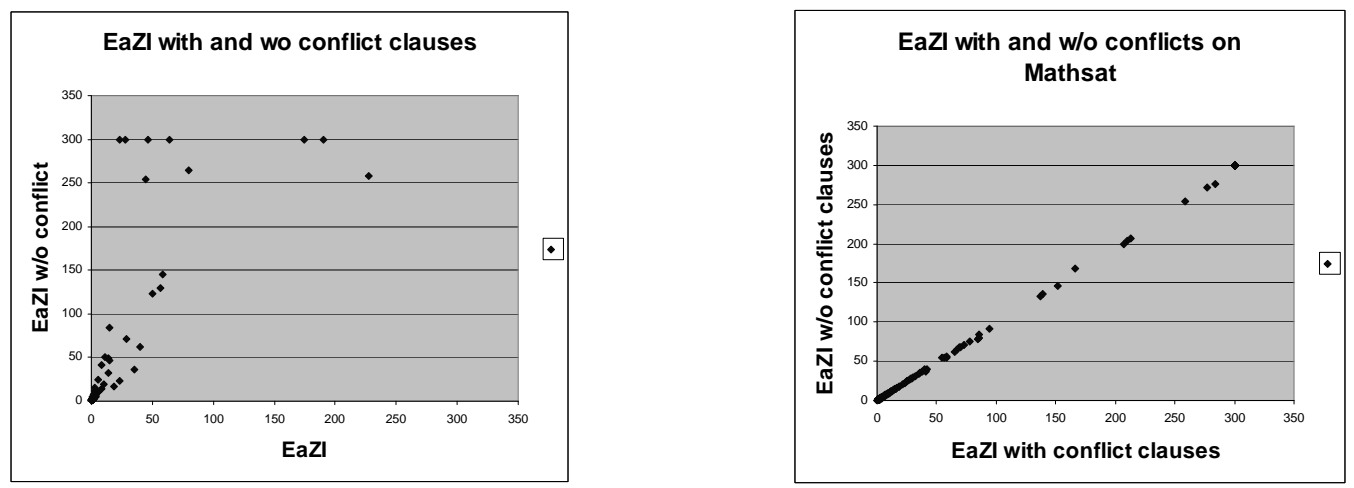

Figure 5. Effect on conflict clauses in Eazi for SAL and Mathsat Benchmarks.

\section{Related Work}

In this section, we compare the interpolant based decision procedure for QFP for other decision procedures for QFP or its restricted fragments. The use of interpolants has been recently explored for finite-state model checking [23] and in refining the abstractions for software verification [16, 19].

Eager approaches for translating a QFP formula to an equisatisfiable Boolean formula employ either the small-model encoding discussed in Section 2.2.2 or add all the theory constraints to the formula [33]. The latter approach can result in an exponential number of constraints being added to the original formula. This translation is often the bottleneck in the method. In [31], the authors encode disjoint set of constraints in a formula using either the small-model encoding or by adding all the theory constraints. The approach was restricted to the difference logic fragment of QFP, and use the number of constraints in the formula to determine the encoding. In contrast, our approach uses small-encoding but increases the size of encoding lazily starting from a small size. Beside, we only add a very small set of theory constraints as conflict clauses in a more lazy manner.

Lazy approaches $[4,13]$ use the lazy proof-explicating framework described in Section 2.2.1. The main bottleneck of these approaches appear to be the large number of invocations of the theory decision procedure. The monomes passed to the decision procedure are often large, even though the reason for unsatisfiability of the monome is often simple and small. Our approach addresses this problem by creating an abstraction of the original formula by using the interpolants and using the lazy approach as a mean to generate conflict clauses. Our experiments indicate that the size of the monome passed to the decision procedure is reduced by more than an order of magnitude and the time spent in the theory decision procedure is reduced considerably. Mathsat [1] use a layered approach, where a sequence of increasingly complete (and therefore more complex) decision procedures 
are used to decide a monome. However, to our knowledge, the approach still suffers from passing large monomes to the linear arithmetic decision procedures.

$\operatorname{DPLL}(\mathrm{T})$ based approaches $[15,25,5]$ use a closer integration of the theory decision procedure with the SAT solver. In addition to the Boolean constraint propagation in the SAT solver, the theory participates in the constraint propagation by adding all the theory facts implied by the theory in the current context. This enables DPLL(T) to detect unsatisfiability earlier than the lazy approaches. However, the decision procedures for the theories become more complex as they need to support the generation of all the facts that are implied by a set of constraints. This may increase the complexity of the ILP decision procedures that already have an exponential worst-case complexity. A promising approach has been suggested by Sheini and Sakallah [32], where they integrate an UTVPI decision procedure in DPLL(T) framework and use the general ILP solver in a lazy framework. Currently, implementations based on DPLL(T) framework are most competitive on the SMT-LIB QFP benchmarks.

The approach closest to our work is Kroening et al.'s [20] work on deciding QFP formulas using Boolean proof of unsatisfiability. Kroening et al. construct an equisatisfiable clausal representation of the original QFP formula by introducing additional variables. The clausal form is encoded to a Boolean formula by performing small-model encoding starting with a small size. An abstraction of the original clausal formula is constructed by choosing a subset of clauses that appear in the proof of unsatisfiability of the Boolean formula. This abstraction is checked using a sound and complete decision procedure for QFP. The sequence is repeated with increasing small-model encoding size. Although similar in many aspects, our approach differ from this work in several ways. First, this method appears to require a clausal representation of the original formula (that can introduce auxiliary variables and destroy some of the structure of the original formula), and the abstraction is limited to be a subset of the clauses in this representation. The abstraction is obtained in a fairly syntactic fashion, by considering the subset of clauses for which there is a corresponding clause in the proof of unsatisfiability for the Boolean formula. We believe that the use of interpolants results in a more semantic method to construct the abstraction. This often results in more concise abstractions being generated. Secondly, unlike their approach we do not require a complete decision procedure for QFP (for checking the abstraction) to ensure the completeness of the procedure. Their experiments (although on a different set of benchmarks) indicate that the lazy decision procedure for ILP dominates the total time of the procedure. Finally, we add conflict clauses from the abstractions generated from smaller domain sizes; this allows us to prune the search space when searching in a larger domain.

\section{Conclusion}

In this paper, we present a framework for using simultaneous under and over approximations to decide a quantifier-free first-order formula. The small-model encoding is used to create the underapproximation of the formula and the interpolant generation from the proofs of unsatisfiability gives an overapproximation of the formula. The use of interpolants provide a semantic and usually a robust way to compute abstractions of an input QFP formula, compared to earlier approach. The method also demonstrates a mechanism to leverage 
some conflict clauses learned during searching in a smaller domain size, to prune the search space during the later search.

\section{References}

[1] G. Audemard, P. Bertoli, A. Cimatti, A. Korniłowicz, and R. Sebastiani. A SAT based approach for solving formulas over boolean and linear mathematical propositions. In International Conference on Automated Deduction (CADE-18), LNCS 2392, pages 195-210, July 2002.

[2] T. Ball, B. Cook, S. K. Lahiri, and L. Zhang. Zapato: Automatic Theorem Proving for Software Predicate Abstraction Refinement. In Computer Aided Verification (CAV '04), LNCS 3114, 2004.

[3] T. Ball, R. Majumdar, T. Millstein, and S. K. Rajamani. Automatic predicate abstraction of C programs. In Programming Language Design and Implementation (PLDI '01), Snowbird, Utah, June, 2001. SIGPLAN Notices, 36(5), May 2001.

[4] C. W. Barrett, D. L. Dill, and A. Stump. Checking Satisfiability of First-Order Formulas by Incremental Translation to SAT. In Proc. Computer-Aided Verification (CAV'02), LNCS 2404, pages 236-249, July 2002.

[5] M. Bozzano, R. Bruttomesso, A. Cimatti, T. A. Junttila, S. Ranise, P. van Rossum, and R. Sebastiani. Efficient satisfiability modulo theories via delayed theory combination. In $C A V$, LNCS 3576, pages 335-349, 2005.

[6] R. E. Bryant, S. K. Lahiri, and S. A. Seshia. Modeling and Verifying Systems using a Logic of Counter Arithmetic with Lambda Expressions and Uninterpreted Functions. In Computer-Aided Verification (CAV'02), LNCS 2404, pages 78-92, July 2002.

[7] T. H. Cormen, C. E. Leiserson, and R. L. Rivest. Introduction to Algorithms. 1990.

[8] W. Craig. Linear reasoning. a new form of the Herbrand-Gentzen theorem. J. Symbolic Logic, 22:250-268, 1957.

[9] M. Davis, G. Logemann, and D. Loveland. A machine program for theorem proving. Communications of the ACM, 5(7):394-397, July 1962.

[10] M. Davis and H. Putnam. A computing procedure for quantification theory. Journal of the ACM, 7(3):201-215, July 1960.

[11] L. M. de Moura and H. Rueß. An experimental evaluation of ground decision procedures. In Computer Aided Verification ( $C A V$ '04), LNCS 3114, pages 162-174. Springer-Verlag, 2004.

[12] D. L. Detlefs, G. Nelson, and J. B. Saxe. Simplify: A theorem prover for program checking. Technical report, HPL-2003-148, 2003. 
[13] C. Flanagan, R. Joshi, X. Ou, and J. Saxe. Theorem Proving using Lazy Proof Explication. In Computer-Aided Verification (CAV 2003), LNCS 2725, pages 355-367, 2003.

[14] C. Flanagan, K. R. M. Leino, M. Lillibridge, G. Nelson, J. B. Saxe, and R. Stata. Extended static checking for java. In ACM SIGPLAN Conference on Programming Language Design and Implementation (PLDI'02), pages 234-245, 2002.

[15] H. Ganzinger, G. Hagen, R. Nieuwenhuis, A. Oliveras, and C. Tinelli. DPLL(T): Fast Decision Procedures. In Computer Aided Verification ( $C A V$ '04), LNCS 3114, pages 175-188, 2004.

[16] T. A. Henzinger, R. Jhala, R. Majumdar, and K. L. McMillan. Abstractions from proofs. In Symposium on Principles of programming languages (POPL '04), pages 232-244, 2004.

[17] ILOG CPLEX. Available at http://ilog.com/products/cplex.

[18] J. Jaffar, M. J. Maher, P. J. Stuckey, and H. C. Yap. Beyond Finite Domains. In Proceedings of the Second International Workshop on Principles and Practice of Constraint Programming, PPCP'94, LNCS 874, pages 86-94, 1994.

[19] R. Jhala and K. L. McMillan. Interpolant-based transition relation approximation. In Computer Aided Verification, 17th International Conference, CAV 2005, LNCS 3576, pages 39-51, 2005.

[20] D. Kroening, J. Ouaknine, S. A. Seshia, and O. Strichman. Abstraction-based satisfiability solving of Presburger arithmetic. In Computer Aided Verification (CAV '04), LNCS 3114, pages 308-320, 2004.

[21] S. K. Lahiri and S. A. Seshia. The UCLID decision procedure. In Computer Aided Verification (CAV'04), LNCS 3114, pages 475-478, 2004.

[22] LP_SOLVE. Available at http://groups.yahoo.com/group/lp_solve/.

[23] K.L. McMillan. Interpolation and sat-based model checking. In CAV 03: ComputerAided Verification, LNCS 2725, pages 1-13. 2003.

[24] M. Moskewicz, C. Madigan, Y. Zhao, L. Zhang, and S. Malik. Chaff: Engineering an efficient SAT solver. In 38th Design Automation Conference (DAC '01), 2001.

[25] R. Nieuwenhuis and A. Oliveras. DPLL(T) with Exhaustive Theory Propagation and its Application to Difference Logic. In Computer Aided Verification, 17th International Conference, CAV 2005, pages 321-334, 2005.

[26] C. H. Papadimitriou. On the complexity of integer programming. J. $A C M, \mathbf{2 8}(4): 765-$ $768,1981$. 
[27] M. Presburger. Uber die Vollstfindigkeit eines gewissea Systems Arithmetic ganzer Zahlen in welchem die Addition als eiilzige Operation hervortritt. In Comptes-rendus du I Congr s de Mathematiciens de Pays Slaves, pages 395:92-101, 1930.

[28] P. Pudl'ak. Lower bounds for resolution and cutting planes proofs and monotone computations. J. of Symbolic Logic, 62(3):981-998, 1995.

[29] H. Rueß and N. Shankar. Solving linear arithmetic constraints. Technical Report CSL-SRI-04-01, SRI International, January 2004.

[30] S. A. Seshia and R. E. Bryant. Deciding quantifier-free Presburger formulas using parameterized solution bounds. In 19th IEEE Symposium of Logic in Computer Science(LICS '04), July 2004.

[31] S. A. Seshia, S. K. Lahiri, and R. E. Bryant. A Hybrid SAT-based Decision Procedure for Separation Logic with Uninterpreted Functions. In Proc. Design Automation Conference (DAC), pages 425-430, June 2003.

[32] H. M. Sheini and K. A. Sakallah. A scalable method for solving satisfiability of integer linear arithmetic logic. In Theory and Applications of Satisfiability Testing, SAT 2005, volume 3569 of $L N C S$, pages 241-256, 2005.

[33] O. Strichman. On solving Presburger and linear arithmetic with SAT. In Formal Methods in Computer-Aided Design (FMCAD '02), LNCS 2517, pages 160-170, 2002.

[34] Zap Project. Available at http://www.research.microsoft.com/tvm.

\section{Appendix A. Termination in the absence of $D_{\max }$}

In the previous section, the termination argument required computing a maximum domain size $D_{\max }$. When $D \geq D_{\max }$, then the formula $\phi_{u}$ is equisatisfiable with $\phi_{b t}$, and therefore also with $\phi$. In this section, we show that we can modify the algorithm slightly to ensure that the computation terminates even in the absence of an upper bound.

The main change required from the previous algorithm is to replace the conflict clause generator $(C C G)$ with a sound and complete lazy SAT-based decision procedure for QFP. Since the conflict clauses generated from the $C C G$ are simply adding more structure to $\phi_{b}$ and not required for completeness, we only required a sound decision procedure QFP for the previous algorithm.

The new algorithm is identical to the previous algorithm except for step 3 (where we do not compute $D_{\max }$ ), step 6 and step 7 . The new step 6 and step 7 becomes (for simplicity, we ignore the conflict clauses $\left.\phi_{c}\right)$ :

- [Overapproximation]. If $\phi_{u}$ is unsatisfiable, compute the Boolean interpolant $\phi_{I}$ of $\phi_{b}$ and $B E\left(\phi_{t h}, D\right)$. We construct the formula $\phi_{o}$ which is an overapproximation of $\phi_{b t}$ as follows:

$$
\phi_{o} \doteq \phi_{I} \wedge \phi_{t h}
$$


We check the satisfiability of $\phi_{o}$ using a lazy SAT-based decision procedure for QFP. If the decision procedure returns UNSATISFIABLE, the algorithm returns UNSATISFIABLE. If the decision procedure returns SATISFIABLE, it also returns $D^{\prime}$, the maximum value of any variable in the satisfying assignment. If $\phi_{I} \Leftrightarrow \phi_{b}$, return SATISFIABLE.

- [Repeat]. Make $D=D^{\prime}$ and go to step 4 .

Lemma 2. For any two distinct iterations $i$ and $j$ of the above algorithm, let $\phi_{I}^{i}$ and $\phi_{I}^{j}$ be the interpolants computed in step 6 of the algorithm in iterations $i$ and $j$ respectively. Then $\phi_{I}^{i} \nLeftarrow \phi_{I}^{j}$.

Proof. Let us assume $\phi_{I}^{i} \Leftrightarrow \phi_{I}^{j}$. Let us assume w.l.o.g. that $i<j$. Let $D_{k}$ and $D_{k}^{\prime}$ be the value of $D$ at the start of the iterations $k$ (for $k \in\{i, j\}$ ). Let us also assume that both the iterations reach step 6. This means that $\phi_{I}^{k} \wedge B E\left(\phi_{t h}, D_{k}\right)$ (for $\left.k \in\{i, j\}\right)$ is unsatisfiable. Moreover $\phi_{I}^{i} \wedge B E\left(\phi_{t h}, D_{i}^{\prime}\right)$ is satisfiable. Since $j>i, D_{j} \geq D_{i}^{\prime}$. Therefore $\phi_{I}^{j} \wedge B E\left(\phi_{t h}, D_{j}\right)$ is clearly satisfiable, and therefore $\phi_{I}^{j}$ can't the interpolant during the iteration $j$. Hence we reach a contradiction.

Theorem 2. The algorithm described in this section that does not precompute $D_{\max }$ is sound and complete for QFP.

Proof. Since there can only be a finite number of distinct Boolean functions over $|B|$ variables and $\phi_{b} \Longrightarrow \phi_{I}$ for any iteration, Lemma 2 ensures that the algorithm above terminates. Therefore, by Theorem 1, the modified algorithm is sound and complete.

In the above algorithm, we have assumed that the decision procedure for QFP used in the overapproximation step (step 6) can construct a satisfying assignment (and therefore provide $D^{\prime}$ ). We can relax this restriction and only increase $D$ by a fixed amount $\delta$ as before and still obtain termination. This is because, using the same idea as Lemma 2, we can ensure that if $\phi_{I}^{i}$ is the interpolant at step $i$, and $j>i+\left(D_{i}^{\prime}-D_{i}\right) / \delta$, then $\phi_{I}^{j}$ is distinct from $\phi_{I}^{i}$. Although this does not allow us to bound the number of iterations (without refering $D_{\max }$ ), we can ensure that in the limit some interpolant $\phi_{I}$ is going to be identical to $\phi_{b}$ for some finite iteration. 